\title{
Relationship Between Bone Density and Salivary Calcium in Women With Rheumatoid Arthritis Undergoing Corticosteroid Therapy
}

\author{
Fateme Arbabi-Kalati, ${ }^{1}$ Mahnaz Sandoghi, ${ }^{2,}{ }^{*}$ Mahsa Moradi, ${ }^{3}$ and Saeede Salimi ${ }^{4}$ \\ ${ }^{1}$ Oral Medicine Department, Oral and Dental Disease Research Center, Zahedan University of Medical Sciences, Zahedan, IR Iran \\ ${ }^{2}$ Medical School, Zahedan University of Medical Sciences, Zahedan, IR Iran \\ ${ }^{3}$ Dentistry student of Zahedan University of Medical Sciences, Zahedan, IR Iran \\ ${ }^{4}$ Medical School, Zahedan University of Medical Sciences, Zahedan, IR Iran \\ "Corresponding author: Mahnaz Sandoghi, Medical School, Zahedan University of Medical Sciences, Zahedan, IR Iran. E-mail: mahnazsandooghi@yahoo.com
}

Received 2016 January 02; Revised 2016 January 30; Accepted 2016 February 18.

\begin{abstract}
Background: Osteoporosis is one of the chronic diseases of the skeletal system characterized by low bone mineral density. Mouth saliva is made up of complex proteins and ions including calcium, potassium, and sodium. Since calcium is an important component of the skeletal system and exists also in saliva, in this study we examined the relationship between salivary calcium and osteoporosis.

Methods: In this analytical cross-sectional study, unstimulated saliva samples were collected from 60 patients with rheumatoid arthritis who had history of steroid use. They were divided into equal groups of osteoporosis, osteopenia, and control. All data were analyzed using ANOVA statistical test in SPSS (v.18).

Results: The average calcium concentration in groups of osteoporosis and osteopenia was $1.7 \pm 0.70$ and $1.7 \pm 0.76$, respectively. Also, the average frequency of dry mouth in osteoporosis and osteopenia groups was 5 and 6, respectively. The observed differences were not statistically significant between the groups $(\mathrm{P}=0.4)$.

Conclusions: According to the present study, there is no significant relationship between salivary and serum calcium and osteoporosis caused by treatment with glucocorticoid.

Keywords: Osteoporosis, Salivary Calcium, Xerostomia
\end{abstract}

\section{Background}

Osteoporosis is one of the chronic diseases of the skeletal system characterized by low bone mineral density resulting in increased bone fragility (1).

Of the factors that increase susceptibility to osteoporosis are menopause, hypothyroidism, rheumatoid arthritis, Cushing's disease, diabetes, use of certain medications such as corticosteroids, Levothyroxine, and hyperthyroidism (2).

Estrogen also reduces available calcium to the bones by increasing urinary excretion of calcium (3). Glucocorticoid induced osteoporosis (GIO) is one of the prevalent and secondary osteoporosis conditions (4). Although this class of drugs is useful in the treatment of inflammatory diseases such as rheumatoid arthritis, autoimmune diseases, and organ transplantation, they can induce damaging effects to bone tissues (5).

As found in studies conducted at cell and tissue level, treatment with this class of drugs may induce little but continuous bone resorption and decrease bone creation, which are caused by apoptosis increase in osteoblasts and osteocytes, extending lifespan of osteoblast (5).
Some studies also showed that the consumption of glucocorticoids will cause bone loss at a rate of $12 \%$ in the first month and then $5 \%-2 \%$ per year. The amount of bone loss depends on 3 parameters: dose of received glucocorticoids per day, duration of use, and type of bone (trabecular or cortical) (5).

The drug is also used for inflammatory diseases. In this case, in addition to adverse effects of the drug on bone, inflammatory diseases will also increase the risk of bone loss and therefore increase the chance of osteoporosis and bone fracture (5).

There are several ways to screen for osteoporosis. Research criterion is bone mineral density (BMD), but methods such as the use of ultrasound waves (QUS) and X-ray (DXA) may also be employed in future. Of course, finding a cheap, easy, and accessible method for early screening of osteoporosis is of importance (6).

Analysis of salivary calcium proposed by Rabiei et al. (7) and Reddy et al. (1) is one of the easiest and safest methods to detect osteoporosis.

Calcium is one of the nutritional keys and essential ingredients for extending bone health. Calcium is one of the essential components of the human body skeleton. Bone 
loss can cause the release of calcium into the blood and then saliva. In this regard, we conducted the current study because of data gap in this field (8).

\section{Methods}

Among rheumatology clinic clients, women with 50 60 years of age with the following criteria were identified: no history of smoking or use of any tobacco product, not being overweight $\left(\mathrm{BMI}<30 \mathrm{~kg} / \mathrm{m}^{2}\right)$, using no medications that cause dry mouth, having no specific systemic diseases affecting salivary glands (such as diabetes, Sjogren's syndrome, etc.), having no oral candidiasis or poor oral hygiene (pockets more than $3 \mathrm{~mm}$ in depth), using no calcium and vitamin D3 supplements, and having no thyroid or parathyroid diseases.

The participants were patients with rheumatoid arthritis who had been under corticosteroid therapy for at least 2 years. Therefore, 22 patients with osteoporosis, 22 patients without osteoporosis, and 22 with osteopenia were recruited and comprised 3 groups of study.

Criterion of determining osteoporosis was based on bone mineral density in spine and hip bone, which was measured as grams per square centimeter.

Bone mineral density was measured by Discorery Hologenic, made in America, based on the T-Score standard of osteoporosis patients. If patient's T-Score is less than 2.5, the patient is considered to have osteoporosis. If T-Score is between -2.5 and -1 , the patient has osteopenia, and if it is > -1 , the patient is considered normal.

The aim of the study was explained to all the participants and written consent was taken from them for participation in the study. The study was approved by the ethics committee of Zahedan University of Medical Sciences.

Unstimulated saliva samples were collected by spitting. Thus, the patients were asked to empty their saliva during 5 minutes, once every minute, into a plastic tube placed at their disposal. After collecting saliva, the saliva flow rate was calculated based on $\mathrm{mL}$ per minute. Saliva was kept at $-20^{\circ} \mathrm{C}$ and after sampling, all the samples were sent to the laboratory to be analyzed.

The women were asked to complete a questionnaire containing a list of symptoms associated with xerostomia (Table 1). If they had 3 positive responses, they assigned to the dry mouth group and those who had no positive responses were placed in the normal group in terms of xerostomia (9).

In this study, in order to measure serum and salivary calcium, we used calcium kits made by Zist Shimi Company in an alkaline environment with the help of Cresolphthalein color reagent that creates an alkaline environment. Then, the resulting color intensity which is proportional
Table 1. The Frequency of Dry Mouth in the Case Groups

\begin{tabular}{lccc}
\hline Variable Group & Present & Not Present & PValue \\
\hline Osteoporosis & 5 & 17 & \\
Osteopenia & 6 & 16 & 0.4 \\
Normal & 6 & 16 & \\
\hline
\end{tabular}

to the amount of calcium in the samples was measured by spectrophotometer at a wavelength of $565 \mathrm{~nm}$ for each patient and then the data were statistically analyzed.

Data were analyzed using SPSS v.18. For comparing calcium concentration between groups, ANOVA test and for comparing dry mouth between groups chi-square test were used (10).

\section{Results}

The results are shown in Tables 2 and 3.

Table 2. Standard Questionnaire for the Possibility of Dry Mouth

\begin{tabular}{|c|c|c|c|}
\hline Rows & Questions & Yes & No \\
\hline 1 & Do you have a dry mouth when eating food? & & \\
\hline 2 & $\begin{array}{l}\text { Do you have trouble when swallowing different } \\
\text { foods? }\end{array}$ & & \\
\hline 3 & $\begin{array}{l}\text { Do you need to drink water when swallowing dry } \\
\text { foods? }\end{array}$ & & \\
\hline 4 & Do you feel any amount of saliva in your mouth? & & \\
\hline 5 & $\begin{array}{l}\text { Do you have a dry mouth at night or upon waking } \\
\text { up? }\end{array}$ & & \\
\hline 6 & Do you have a dry mouth when going on trips? & & \\
\hline 7 & $\begin{array}{l}\text { Do you use chewing gums or chocolates for } \\
\text { eliminating your dry mouth feeling? }\end{array}$ & & \\
\hline 8 & $\begin{array}{l}\text { Do you ever wake up during the night because of } \\
\text { thirst? }\end{array}$ & & \\
\hline 9 & Do you have trouble when tasting foods? & & \\
\hline 10 & Do you have a burning feeling in your tongue? & & \\
\hline
\end{tabular}

Table 3. Average Serum Calcium, Salivary Calcium, and Patients' Age in the Case Groups

\begin{tabular}{lccc}
\hline \multirow{2}{*}{ Group } & \multicolumn{3}{c}{ Variable } \\
\cline { 2 - 4 } & Salivary Calcium & Serum Calcium & Age \\
\hline Osteoporosis & $1.7 \pm 0.7$ & $8.9 \pm 1.8$ & $55.3 \pm 5.5$ \\
Osteopenia & $1.7 \pm 0.67$ & $9.1 \pm 1$ & $52.2 \pm 5.6$ \\
Normal & $1.7 \pm 0.74$ & $9 \pm 1.7$ & $51.1 \pm 5.2$ \\
PValue & 0.9 & 0.9 & 0.8 \\
\hline
\end{tabular}


Table 2 shows average serum calcium, salivary calcium, and patients' age in the study groups. As can be seen, there is no statistically significant difference between the groups.

Table 3 shows the frequency of dry mouth in the case groups indicating that there is no significant difference between the groups.

\section{Discussion}

Osteoporosis is an asymptomatic disease, which is probably one of the most common progressive bone diseases. Osteoporosis is a chronic disease of the skeletal system characterized by low bone mass and increased bone fragility. One of the most important problems associated with this disease is inability to detect until bones are weakened and bone damages occur (8). Thus, identifying individuals who are at greater risk of developing this illness for various reasons is of great importance.

The gold standard for detecting the disease is determining bone mineral density (BMD), which is a test with high sensitivity and specificity and high cost, as well (11).

Another proposed method is the analysis of saliva, because saliva can be used as an indicator of the body status $(11,12)$.

In the current study, salivary calcium was compared between women under glucocorticoid therapy in two groups with osteoporosis and osteopenia and women in the control group. Also, these two groups were compared with the control group in terms of dry mouth.

In the present study, no significant correlation was found between average salivary calcium concentrations in the case groups and those of the control group.

In a study conducted by Hattori et al. in 2014, it was tried to examine the relationship between salivary calcium concentration and changes of bone mineral density associated with estrogen deficiency or differences in the uptake of calcium in female rats. They attempted to compare calcium concentration and BMD in both Sham-Operated and ovariectomized groups. It was also observed that the BMD was significantly lower in the ovariectomized group. However, there was no significant relationship between the two groups in calcium concentration (3). In the same study, 42 rats were divided after ovariectomy into groups of five and received calcium supplements at doses of 0.01, 0.1, 0.6, 1.2, 2.4\%. It was observed that BMD was significantly lower in the first two groups than the other groups. However, in terms of calcium concentration, there was no significant difference between the groups, which is similar to our findings.

In a study carried out by Ansari Moghaddam et al. in 2016, the relationship between periodontitis and osteo- porosis was investigated with the help of salivary biomarkers. They divided 60 people selected from clients of dental schools into two groups of 30 including a control group and a group of patients with severe chronic periodontitis. Patients of the case group were completely healthy in terms of systemic osteoporosis-inducing diseases such as rheumatism, malnutrition, Cushing, and hyperparathyroidism. They were all non-smokers and they had no history of taking steroids or immunosuppressive (11). In this study, they did not observe a significant correlation between salivary calcium and phosphorus concentrations and BMD level between the case and control groups which was similar to our study findings. They also stated that the use of these biomarkers for detection of osteoporosis is not a good criterion for identifying osteoporosis and proposed larger sample size in future research (11).

But unlike the current study, in some studies, there was no significant relationship between salivary calcium concentration and BMD in patients. In a study conducted in 2008 by Reddy et al., the signs of mouth, salivary calcium, phosphorus, alkaline phosphatase, and dental x-ray changes were examined in postmenopausal women with osteoporosis and osteopenia compared to healthy subjects (1). They concluded that the mean age was significantly higher in groups with osteoporosis and osteopenia than the control group. Moreover, the average salivary calcium, phosphorus, and alkaline phosphatase was higher in the two case groups than the control group, which is not consistent with our study results (1).

In a study by Rabiei et al. in 2013,40 postmenopausal female subjects with osteoporosis and 40 postmenopausal female subjects without osteoporosis were studied. It was found that there is a significant relationship between menopause period and the level of calcium; also, women who were menopause over 16 years were more at risk of osteoporosis (7).

Dry mouth (DM) or Xerostomia is among common complaints in the elderly, which can result in oral and oropharyngeal inconveniences and reduced quality of life and increased pain (10). DM can occur for various reasons such as getting some of systemic diseases like diabetes and Sjogren's syndrome, reduced liquid intake, central cognitive changes, and oral sensory disturbances as well as disturbed mental state (13).

Dry mouth can also occur due to changes in salivary composition in menopausal women. In previous studies, it has been suggested that reduced levels of salivary estrogen and progesterone and increased cortisol and parotid hormone (PTH) in the blood and saliva are associated with dry mouth. On the other hand, the effects of estrogen, cortisol, and PTH on bone Turn Over and decrease of bone density are expected in patients (14). 
In the current study no significant difference was found between patients with osteoporosis and osteopenia undergoing glucocorticoid therapy and the control group in terms of xerostomia $(\mathrm{P}=0.4)$.

Of the studies conducted on dry mouth, we can point out a study in 2011 by Agha Hosseini et al. in which they attempted to examine the relationship between dry mouth and bone density at lumbar spine area. They concluded that the average BMD at Lumbar Spine area was significantly lower in the case group than the control group, and there was a significant inverse correlation between BMD and degree of dry mouth, which was not consistent with our results (9).

In a study conducted in 2013 by Agha Hosseini et al. they compared the flow of saliva, serum and salivary testosterone, and femur bone mineral density in women with and without dry mouth feeling. In this case-control study, they only recruited postmenopausal women (14). To determine the severity of dry mouth feeling, patients' responses to the questionnaires of the other case groups were graded and ultimately each person's severity of dry mouth was determined (14). The study showed that low salivary flow, low femur bone density, and increased stimulated salivary testosterone are related to an increased feeling of xerostomia in post-menopausal women and concluded that among the mentioned factors, reduced flow of saliva is probably the most important factor causing dry mouth (14).

Thus, according to the previous animal and human studies, we can state that one of the possible reasons for the lack of a significant difference between case and control groups in terms of salivary calcium found in our study is because the study subjects had systemic inflammatory diseases and took different doses of glucocorticoid. Therefore, drug-induced osteoporosis may have a different trend than osteoporosis in postmenopausal patients with complete general health.

\subsection{Conclusion}

Based on the present results, there is no significant relationship between salivary and serum calcium and osteoporosis caused by treatment with glucocorticoid, and we cannot use these markers to predict osteoporosis. Therefore, it is suggested that researchers conduct studies with larger sample sizes or examine postmenopausal patients with osteoporosis suffering from any systemic disease.

\section{References}

1. Reddy S, Karthikeyan R, Sherlin HJ, Anuja N, Pratibha R, Priya P, et al. Oral signs and salivary parameters as indicators of possible osteoporosis and osteopenia in postmenopausal women-A study of $45 \mathrm{sub}$ jects. Brazil J Oral Sci. 2016;7(24):1502-6.

2. Lango D, Fanci A, Hanser S, Jamson J, Lascalzo J. In: Harrison International Disease. Lango D, Fanci A, Hanser S, Jamson J, Lascalzo J, editors. ; 1995. Iron Deficiency And Other Hyper Prolifrative Anemias.

3. Hattori S, Agata U, Park JH, Iimura Y, Tokuda S, Ezawa I, et al. The relationship between salivary calcium concentration and differences in bone mineral density level in female rats. J Nutr Sci Vitaminol (Tokyo). 2014;60(3):152-8. [PubMed: 25078370].

4. Hansen KE, Kleker B, Safdar N, Bartels CM. A systematic review and meta-analysis of glucocorticoid-induced osteoporosis in children. Seminars Arthritis Rheumatism. 2014;44(1):47-54.

5. Leib ES, Winzenrieth R. Bone status in glucocorticoid-treated men and women. Osteoporos Int. 2016;27(1):39-48. doi: 10.1007/s00198-0153211-1. [PubMed: 26252975].

6. Boonen S, Nijs J, Borghs H, Peeters H, Vanderschueren D, Luyten FP. Identifying postmenopausal women with osteoporosis by calcaneal ultrasound, metacarpal digital X-ray radiogrammetry and phalangeal radiographic absorptiometry: a comparative study. Osteoporos Int. 2005;16(1):93-100. doi: 10.1007/s00198-004-1660-z. [PubMed: 15197540].

7. Rabiei M, Masooleh IS, Leyli EK, Nikoukar LR. Salivary calcium concentration as a screening tool for postmenopausal osteoporosis. Int J Rheum Dis. 2013;16(2):198-202. doi: 10.1111/1756-185X.12003. [PubMed: 23773645].

8. Sahithi D, Reddy S, Rajesh N, Priyadarshini S, Krishnan M, Upendra G. Post menopause and Osteoporosis - Unfolding the link. Int J Med. 2015;3(1):26. doi: 10.14419/ijm.v3i1.4266.

9. Agha-Hosseini F, Mirzaii-Dizgah I, Moosavi MS. Relationship of lumbar spine bone mineral density and oral dryness feeling in menopause. Menopause. 2011;18(6):625-8. doi: 10.1097/gme.0b013e31820285b2. [PubMed: 21358351].

10. Sewon L, Laine M, Karjalainen S, Leimola-Virtanen R, Hiidenkari T, Helenius $H$. The effect of hormone replacement therapy on salivary calcium concentrations in menopausal women. Arch Oral Biol. 2000;45(3):201-6. [PubMed: 10761873].

11. Moghadam SA, Zakeri Z, Fakour SR, Moghaddam AA. Does Salivary Calcium and Phosphate Concentrations Adequately Reflect Bone Mineral Density in Patients with Chronic Periodontitis? Glob J Health Sci. 2016;8(10):56723. doi: 10.5539/gjhs.v8n10p282. [PubMed: 27302461].

12. Agha-Hosseini F, Mirzaii-Dizgah I, Moosavi MS. Relationship of serum and saliva calcium, phosphorus and alkaline phosphatase with dry mouth feeling in menopause. Gerodontology. 2012;29(2):e1092-7. doi: 10.1111/j.1741-2358.2012.00619.x. [PubMed: 22260185].

13. Martin S, Greenberg M, Glick M, Ship J. Burket's oral medicine. Hamilton: BC Decker; 2008.

14. Agha-Hosseini F, Moosavi MS, Mirzaii-Dizgah I. Salivary flow, testosterone, and femur bone mineral density in menopausal women with oral dryness feeling. Oral Surg Oral Med Oral Pathol Oral Radiol. 2013;115(5):612-6. doi: 10.1016/j.0ooo.2012.11.014. [PubMed: 23433570]. 